\title{
Latest Results on Few-Body Physics from $\mathrm{HI} \gamma$
}

\author{
Haiyan Gao* \\ Department of Physics, Duke University and Triangle Universities Nuclear Laboratory \\ E-mail: gao@phy.duke.edu
}

\section{Georgios Laskaris ${ }^{\dagger}$}

Department of Physics, Duke University and Triangle Universities Nuclear Laboratory

E-mail: laskarisestanford.edu

\section{Xuefei Yan}

Department of Physics, Duke University and Triangle Universities Nuclear Laboratory

E-mail: xy33@phy.duke.edu

In this talk we discuss the recently completed and upcoming experiments on few-nucleon targets at the High Intensity $\gamma$-ray Source $(\mathrm{HI} \gamma \mathrm{S})$ facility located at Triangle Universities Nuclear Laboratory (TUNL). Several experiments took place or are planned in order to measure the GerasimovDrell-Hearn sum rule integrand on various light nuclei below pion production threshold and extract information on the electromagnetic structure and spin polarizabilities of the nucleon. In this paper we focus more on the photodisintegration and Compton scattering experiments on polarized ${ }^{3} \mathrm{He}$ at the HI $\gamma \mathrm{S}$ facility.

The 8th International Workshop on Chiral Dynamics, CD2015 ***

29 June 2015 - 03 July 2015

Pisa,Italy

\footnotetext{
* Speaker.

${ }^{\dagger}$ Currently at Stanford University, Stanford CA, 94305, USA.
} 


\section{Photodisintegration of ${ }^{3}$ He with Double Polarizations}

An important window for the study of QCD is through the investigation of the structure and particularly the spin structure of the nucleon and few-body nuclei. Therefore sum rules involving the spin structure of the nucleon or nuclei are at the forefront of intensive experimental and theoretical efforts. Among sum rules, the GDH sum rule [1] is particularly interesting. This sum rule relates the energy-weighted difference of the spin-dependent total photo-absorption cross sections $\sigma^{P}\left(\sigma^{A}\right)$ for target spin and beam helicity parallel (antiparallel) to static properties of the target nucleon/nucleus, i.e. the anomalous magnetic moment and the mass, as follows:

$$
I^{G D H}=\int_{v_{t h r}}^{\infty}\left(\sigma^{P}-\sigma^{A}\right) \frac{d v}{v}=\frac{4 \pi^{2} \alpha}{M^{2}} \kappa^{2} I,
$$

where $v$ is the photon energy, $v_{t h r}$ is the pion production/photodisintegration threshold on the nucleon/nucleus, $\kappa$ is the anomalous magnetic moment, $M$ is the mass and $I$ is the spin of the nucleon/nucleus. There have been worldwide efforts in testing the GDH sum rule on proton and neutron [2,3]. More recently, experimental investigations of the GDH sum rule on nuclei such as the deuteron $[4,5,6]$ and ${ }^{3} \mathrm{He}[7,8,9,10]$ have begun at $\mathrm{HI} \gamma \mathrm{S}$ facility and elsewhere.

The determination of the GDH sum rule on ${ }^{3} \mathrm{He}$ at the energy region between the two-body photodisintegration $(\sim 5.5 \mathrm{MeV})$ and the pion production threshold $(\sim 140 \mathrm{MeV})$ is particularly interesting for a number of reasons. This energy region has an important contribution to the overall sum rule $[8,11]$ and it is a region where one can test state-of-the-art three-body calculations.

A polarized ${ }^{3} \mathrm{He}$ target is commonly used as a polarized neutron target to extract the electromagnetic form factors [12, 13, 14] and the spin structure functions [15] of the neutron since the nuclear spin of ${ }^{3} \mathrm{He}$ is carried mostly by the unpaired neutron. To acquire information about the neutron using a polarized ${ }^{3} \mathrm{He}$ target, nuclear corrections relying on three-body calculations need to be used, but first they must be validated by experiments. The experimental determination of the GDH integral on ${ }^{3} \mathrm{He}$ can also test to what extent a polarized ${ }^{3} \mathrm{He}$ target is an effective polarized neutron target.

The GDH integral below pion threshold can be estimated based on two sets of three-body calculations which are performed mainly through the machinery of Faddeev [17] and Alt-GrassbergerSandhas equations (AGS) [18] and have been carried out for both two-body and three-body photodisintegration of ${ }^{3} \mathrm{He}$ with double polarizations. These calculations $[19,20]$ use a variety of nucleon-nucleon (NN) potentials like Argonne V18 (AV18) [21] or CD Bonn [22, 23] and threenucleon forces (3NFs) like Urbana IX (UIX) [24] or CD Bonn $+\Delta$ [19], with the latter yielding an effective $3 \mathrm{NF}$ through the $\Delta$-isobar excitation. The maximum plateau value that both sets of calculations [19] ([20]) predict for the GDH integral of ${ }^{3} \mathrm{He}$ below pion threshold is $\sim 140 \mu \mathrm{b}$ [8] which equals to the sum of the contributions from the three-body $\sim 170 \mu \mathrm{b}(\sim 130 \mu \mathrm{b})$ and the two-body $\sim-30 \mu \mathrm{b}(\sim 10 \mu \mathrm{b})$ components.

\subsection{The Experiments}

\section{Measurement of ${ }^{3} \vec{H} e(\vec{\gamma}, n) p p$ at $\mathbf{E}_{\gamma}=\mathbf{1 2 . 8}, 14.7$ and $16.5 \mathrm{MeV}$}

The experiments $[7,8]$ on the three-body photodisintegration of ${ }^{3} \mathrm{He}$ using a longitudinally polarized ${ }^{3} \mathrm{He}$ target and a circularly polarized $\gamma$-ray beam took place at the $\mathrm{HI} \gamma \mathrm{S}$ facility [25] 
of TUNL at the incident photon energies of $12.8,14.7$ and $16.5 \mathrm{MeV}$. A nearly mono-energetic, $\sim 100 \%$ circularly-polarized pulsed $\gamma$-ray beam was used. The beam was collimated using a $12 \mathrm{~mm}$ diameter collimator resulting in on-target intensities of $(0.7-2.0) \times 10^{8} \gamma / \mathrm{s}$ and an energy spread of $\Delta v / v \sim 3.0-5.0 \%$. The on-target intensity of the beam was determined using the well-known $\mathrm{d}(\gamma, \mathrm{n}) \mathrm{p}$ cross section [26] and two BC501A liquid scintillator neutron detectors mounted at a scattering angle of $90^{\circ}$ downstream of the ${ }^{3} \mathrm{He}$ target.

Upstream of the flux monitor, the polarized $\gamma$-beam was incident on a polarized ${ }^{3} \mathrm{He}$ cell. The ${ }^{3} \mathrm{He}$ target, named SPOT, used for all experiments both on two- and three-body photodisintegration is a single piece of pyrex glassware coated with a thin layer of GE180 glass. The ${ }^{3} \mathrm{He}$ density of SPOT is $6.4 \pm 0.2 \mathrm{amg}$. A $\mathrm{N}_{2}$ reference cell was used for background subtraction. Details concerning their technical characteristics and the spin exchange optical pumping technique used to polarize the ${ }^{3} \mathrm{He}$ target can be found in $[7,8,27,28,29]$. In order to form the parallel (antiparallel) configurations of the ${ }^{3} \mathrm{He}$ spin with the beam helicity, and extract the spin-dependent cross sections and the GDH integrand, $\left(\sigma^{P}-\sigma^{A}\right) / \nu$, the spin of the target was flipped every 15 mins during all experiments. This time interval was small enough to suppress relevant systematic uncertainties. The polarization of the ${ }^{3} \mathrm{He}$ target was measured using the nuclear magnetic resonance-adiabatic fast passage (NMR-AFP) [30] technique calibrated by electron paramagnetic resonance (EPR) method [31]. ${ }^{3} \mathrm{He}$ target polarization was found to be between $33 \%$ and $45 \%$.

An array of sixteen liquid scintillator BC-501A counters was used to detect the neutrons from the ${ }^{3} \vec{H} e(\vec{\gamma}, n) p p$ reaction. The detectors were placed at the horizontal plane every $15^{\circ}$, symmetrically on each side of the beam, at laboratory scattering angles from $30^{\circ}$ to $165^{\circ}$. No detectors were placed at the laboratory angles between $60^{\circ}$ and $120^{\circ}$ due to the proximity to a pair of Helmholtz coils which provided the holding field for the polarized ${ }^{3} \mathrm{He}$ target.

Three quantities were recorded for each event: the pulse height (PH), the time-of-flight (TOF) and the pulse shape discrimination (PSD) signals. Initially, a PH cut was applied at $0.162 \mathrm{MeV}_{e e}{ }^{1}$ to set the detector threshold. The correlations between the PSD, PH and TOF were utilized to extract the neutron events and to remove the $\gamma$-ray events and two-dimensional cuts were applied on these histograms. The same cuts were used for the data taken with the $\mathrm{N}_{2}$ reference cell to subtract the background contributions. The outgoing neutron energy was determined using the measured TOF of the neutrons assuming they were produced from the center of the ${ }^{3} \mathrm{He}$ target cell. More details about this analysis can be found in [7, 8].

The spin-dependent double-, single-differential and total cross sections, and the contributions of the three-body photodisintegration of ${ }^{3} \mathrm{He}$ to the GDH integrand were obtained $[7,8]$ and compared with the state-of the-art three-body calculations $[19,20]$ at all incident energies. Although a very good agreement was observed between the measurements $[7,8]$ and the calculations based on [19] at 12.8 and $14.7 \mathrm{MeV}$, a difference between the measured GDH integrand and the calculations was found at the incident photon energy of $16.5 \mathrm{MeV}$. The measured GDH integrand at 16.5 $\mathrm{MeV}$ was found to be slightly more than one standard deviation larger than the maximum calculated value based on [19].

To investigate whether the larger than expected GDH integrand value at $16.5 \mathrm{MeV}$ is due to statistics and to further quantify the three-body contribution to the GDH integral, measurements

\footnotetext{
${ }^{1}$ One $\mathrm{MeV}_{e e}$ is the amount of light energy generated by an electron having kinetic energy of $1 \mathrm{MeV}$.
} 
above $16.5 \mathrm{MeV}$ for the three-body photodisintegration channel are necessary. These measurements combined with the recently acquired data from the two-body photodisintegration channel [8] will constrain the contribution to the GDH integral for ${ }^{3} \mathrm{He}$ below the pion-threshold.

\section{Measurement of ${ }^{3} \vec{H} e(\vec{\gamma}, p) D$ at $\mathbf{E}_{\gamma}=29.0 \mathrm{MeV}$}

The first measurement on the two-body photodisintegration of ${ }^{3} \mathrm{He}$ with double polarizations at incident photon energy of $29.0 \mathrm{MeV}$ was performed in May 2014. The nearly monoenergetic, $\sim 100 \%$ circularly-polarized $\gamma$-ray beam of $\mathrm{HI} \gamma \mathrm{S}$ was used and almost two hundred hours of production data were acquired on both ${ }^{3} \mathrm{He}$ and $\mathrm{N}_{2}$ reference cells. The protons from the two-body photodisintegration of polarized ${ }^{3} \mathrm{He}$ were detected by 72 fully depleted silicon detectors placed every $25^{\circ}$ at proton scattering angles between $45^{\circ}$ and $120^{\circ}$ degrees (18 detectors at each angle). Six aluminum hemispheres were used to place the detectors $\sim 10 \mathrm{~cm}$ away from the center of the ${ }^{3} \mathrm{He}$ target chamber (three on each side of the cell facing each other). Each hemisphere housed up to twelve detectors and allowed to maximize the number of detectors around the ${ }^{3} \mathrm{He}$ cell.

As in the case of the three-body photodisintegration experiments, the spin exchange optical pumping [27] technique was used to polarize the ${ }^{3} \mathrm{He}$ target named SPOT and the NMR-AFP [30] and EPR [31] techniques were used to measure its polarization.
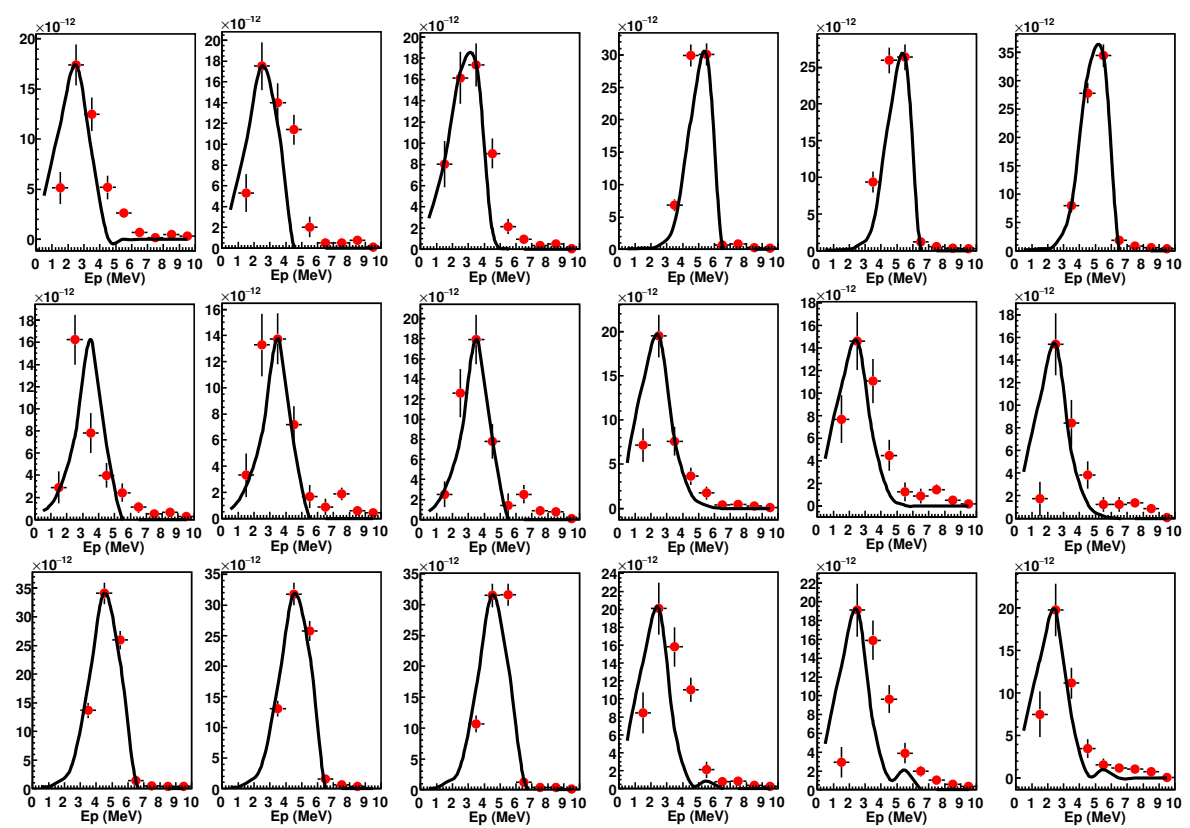

Figure 1: The experimental proton yield spectra (red markers) in comparison to the spectra simulated using as input the calculations (black curves) provided by Deltuva $\mathrm{et}$ al. for all detectors at the proton scattering angle of $95^{\circ}$. The thickness of the glass in the simulation is adjusted in order to match the location of the proton energy peaks and an almost perfect agreement is observed at all angles. The proton energy bin width is $1.0 \mathrm{MeV}$.

Fig. 1 shows the extracted proton spectra at the scattering angle of $95^{\circ}$ divided by the integrated photon flux compared with the GEANT4 [32] simulated spectra using as input the calculations of Deltuva et al. Unlike the three-body photodisintegration where the neutrons passed through the wall of the target chamber of the ${ }^{3} \mathrm{He}$ cell mostly unaffected, in the case of two-body phtodisinte- 
gration the glass thickness is of utmost importance for the precise simulation of the energy loss of the protons in the glass. The glass thickness implemented in the simulation is modified accordingly and always within the range dictated by measurements taken throughout the ${ }^{3} \mathrm{He}$ and $\mathrm{N}_{2}$ reference cells at the University of Virginia. The same procedure needs to be repeated for both ${ }^{3} \mathrm{He}$ and $\mathrm{N}_{2}$ reference cells until all simulated spectra at all proton scattering angles match the shape of the observed experimental proton yield spectra.

The data analysis is currently in progress and quantities such as spin-dependent single-differential and total cross sections and the ${ }^{3} \mathrm{He}$ GDH integrand value from the two-body break-up at $29.0 \mathrm{MeV}$ will be extracted soon. The extension of these measurements to higher photon energies and the performance of measurements on three-body breakup channel above $16.5 \mathrm{MeV}$ will provide crucial tests of the differential cross sections, the energy dependence of the predictions, and whether the contribution to the GDH integral is indeed dominated by the three-body channel below the pion threshold. These measurements, when combined with data above pion threshold from other laboratories, will directly test the ${ }^{3} \mathrm{He}$ GDH sum rule prediction.

\section{Compton Scattering on ${ }^{3} \mathrm{He}$ nuclei with Double Polarizations}

Neutrons and protons (nucleons) are basic building blocks of the visible matter in the universe. To understand the internal structures of nucleons, probes of electrons, photons, etc, have been used in various experiments. The response of nucleons to an external electromagnetic field has been studied and parameterized by quantities called polarizabilities. The spin-independent response of nucleons to an external electromagnetic field can be parameterized by electromagnetic polarizabilities $\alpha$ and $\beta$, while the spin-dependent ones are parameterized by spin polarizabilities $\gamma_{1}, \gamma_{2}, \gamma_{3}$ and $\gamma_{4}$ [33]. The physics picture of the polarizabilities has been discussed in more detail in [33, 34].

One common way to describe the behavior of nucleons in an external electromagnetic (EM) field is to treat the EM field quantized as photons, and expand the Hamiltonian of interaction between the nucleon and the EM field in orders of power of the photon energy $(\omega)[34,35]$. Electric field $(\vec{E})$ and magnetic field $(\vec{B})$ are of order 1, because they involve spatial and time derivatives of the four-vector potential field $\left(A^{\mu}\right)$, while $A^{\mu}$ is defined to be of order 0 . The general amplitude for Compton scattering can be derived from the Hamiltonian. Electromagnetic polarizabilities $\alpha$ and $\beta$ are parameters for the second order terms. Combinations of spin polarizabilities $\gamma_{1}, \gamma_{2}, \gamma_{3}$ and $\gamma_{4}$ appear as parameters for the third order terms. The forward and backward spin polarizabilities $\left(\gamma_{0}=\gamma_{1}-\gamma_{2}-2 \gamma_{4}\right.$ and $\gamma_{\pi}=\gamma_{1}+\gamma_{2}+2 \gamma_{4}$, respectively) are also employed in experiments and theories.

Expansion of the Hamiltonian up to the third order of the photon energy involves both electromagnetic and spin polarizabilities[34, 35, 36, 37]:

$$
\begin{aligned}
H= & \frac{(\vec{p}-Z e \vec{A})^{2}}{2 M}+Z e \phi-\vec{\mu} \cdot \vec{B}-2 \pi\left[\alpha \vec{E}^{2}+\beta \vec{B}^{2}\right] \\
& -2 \pi\left[\gamma_{E_{1} E_{1}} \vec{\sigma} \cdot(\vec{E} \times \dot{\vec{E}})+\gamma_{M_{1} M_{1}} \vec{\sigma} \cdot(\vec{B} \times \dot{\vec{B}})-2 \gamma_{M_{1} E_{2}} \sigma_{i} E_{i j} B_{j}+2 \gamma_{E_{1} M_{2}} \sigma_{i} B_{i j} E_{j}\right]
\end{aligned}
$$

where $\vec{p}$ stands for momentum of the particle (nucleon in this case), $\vec{A}$ for vector potential of the EM field, $e$ for the unit electric charge, $Z$ for number of unit charges of the nucleon, $\phi$ for electric 
potential from the EM field, $\vec{\sigma}$ for the spin and $\vec{\mu}$ for the magnetic dipole moment of the nucleon, $\vec{B}$ for the magnetic field, $\vec{E}$ for the electric field. $\alpha$ and $\beta$ are electromagnetic polarizabilities. $E_{i j}=\frac{1}{2}\left(\partial_{i} E_{j}+\partial_{j} E_{i}\right), B_{i j}=\frac{1}{2}\left(\partial_{i} B_{j}+\partial_{j} B_{i}\right)$ and summation over repeated index is assumed. $\gamma_{E_{1} E_{1}}=-\gamma_{1}-\gamma_{3}, \gamma_{M_{1} M_{1}}=\gamma_{4}, \gamma_{M_{1} E_{2}}=\gamma_{2}+\gamma_{4}$ and $\gamma_{E_{1} M_{2}}=\gamma_{3}[35]$.

The scattering amplitude can be calculated based on Eq. (1) [34] as:

$$
\begin{aligned}
T(\omega, z)= & A_{1}(\omega, z)\left(\vec{\varepsilon} \cdot \vec{\varepsilon}^{*^{\prime}}\right)+A_{2}(\omega, z)\left(\vec{\varepsilon}^{\prime} \cdot \hat{k}\right)\left(\vec{\varepsilon} \cdot \vec{k}^{\prime}\right) \\
& +i A_{3}(\omega, z) \vec{\sigma} \cdot\left(\vec{\varepsilon}^{*^{\prime}} \times \vec{\varepsilon}\right)+i A_{4}(\omega, z) \vec{\sigma} \cdot\left(\hat{k}^{\prime} \times \hat{k}\right)\left(\vec{\varepsilon}^{\prime} \cdot \vec{\varepsilon}\right) \\
& +i A_{5}(\omega, z) \vec{\sigma} \cdot\left[\left(\vec{\varepsilon}^{\prime} \times \hat{k}\right)\left(\vec{\varepsilon} \cdot \hat{k}^{\prime}\right)-\left(\vec{\varepsilon} \times \hat{k}^{\prime}\right)\left(\vec{\varepsilon}^{\prime} \cdot \hat{k}\right)\right] \\
& +i A_{6}(\omega, z) \vec{\sigma} \cdot\left[\left(\vec{\varepsilon}^{*^{\prime}} \times \hat{k}^{\prime}\right)\left(\vec{\varepsilon} \cdot \hat{k}^{\prime}\right)-(\vec{\varepsilon} \times \hat{k})\left(\vec{\varepsilon}^{*^{\prime}} \cdot \hat{k}\right)\right]
\end{aligned}
$$

where

$$
\begin{aligned}
& A_{1}(\omega, z)=-\frac{Z^{2} e^{2}}{M_{N}}+\frac{e^{2} \omega^{2}}{4 M_{N}^{3}}\left((Z+\kappa)^{2}(1+z)-Z^{2}\right)(1-z)+4 \pi \omega^{2}\left(\alpha_{E 1}+z \beta_{M 1}\right) \\
& A_{2}(\omega, z)=\frac{e^{2} \omega^{2}}{4 M_{N}^{3}} \kappa(2 Z+\kappa) z-4 \pi \omega^{2} \beta_{M 1} \\
& A_{3}(\omega, z)=\frac{e^{2} \omega}{2 M_{N}^{2}}\left(Z(Z+2 \kappa)-(Z+\kappa)^{2} z\right)+A_{3}^{\pi^{0}}+4 \pi \omega^{3}\left(\gamma_{1}-\left(\gamma_{2}+2 \gamma_{4}\right) z\right) \\
& A_{4}(\omega, z)=-\frac{e^{2} \omega}{2 M_{N}^{2}}(Z+\kappa)^{2}+4 \pi \omega^{3} \gamma_{2} \\
& A_{5}(\omega, z)=\frac{e^{2} \omega}{2 M_{N}^{2}}(Z+\kappa)^{2}+A_{5}^{\pi^{0}}+4 \pi \omega^{3} \gamma_{4} \\
& A_{6}(\omega, z)=-\frac{e^{2} \omega}{2 M_{N}^{2}} Z(Z+\kappa)+A_{6}^{\pi^{0}}+4 \pi \omega^{3} \gamma_{3}
\end{aligned}
$$

and $Z$ is the number of unit charge $e$ of the nucleon, $\kappa$ is the anomalous magnetic moment of the nucleon, $M_{N}$ is the mass of the nucleon, $\omega$ is the photon energy, $z$ is the cosine of the scattering angle $\theta$. The $\vec{\varepsilon}$ and $\vec{\varepsilon}^{\prime}$ are the polarizations of incoming and outgoing photons, respectively. The terms $A_{3}^{\pi^{0}}, A_{5}^{\pi^{0}}, A_{6}^{\pi^{0}}$ are contributions from $\pi^{0}$ pole. The details of $\pi^{0}$ pole contributions are discussed in $[34,35]$.

More details of the theoretical basis and some of the previous experimental extractions of the polarizabilities are presented in [34]. The newest data measuring the electromagnetic polarizabilities of neutron using the Baldin sum rule was published in [38]:

$$
\begin{aligned}
& \alpha_{n}=[11.55 \pm 1.25(\text { stat }) \pm 0.2(B S R) \pm 0.8(t h)] \times 10^{-4} \mathrm{fm}^{3} \\
& \beta_{n}=[3.65 \mp 1.25(\text { stat }) \pm 0.2(B S R) \mp 0.8(t h)] \times 10^{-4} \mathrm{fm}^{3}
\end{aligned}
$$

The current world data for electromagnetic polarizabilities $\alpha$ and $\beta$ of proton and neutron are shown in Fig. 2 [39]. 


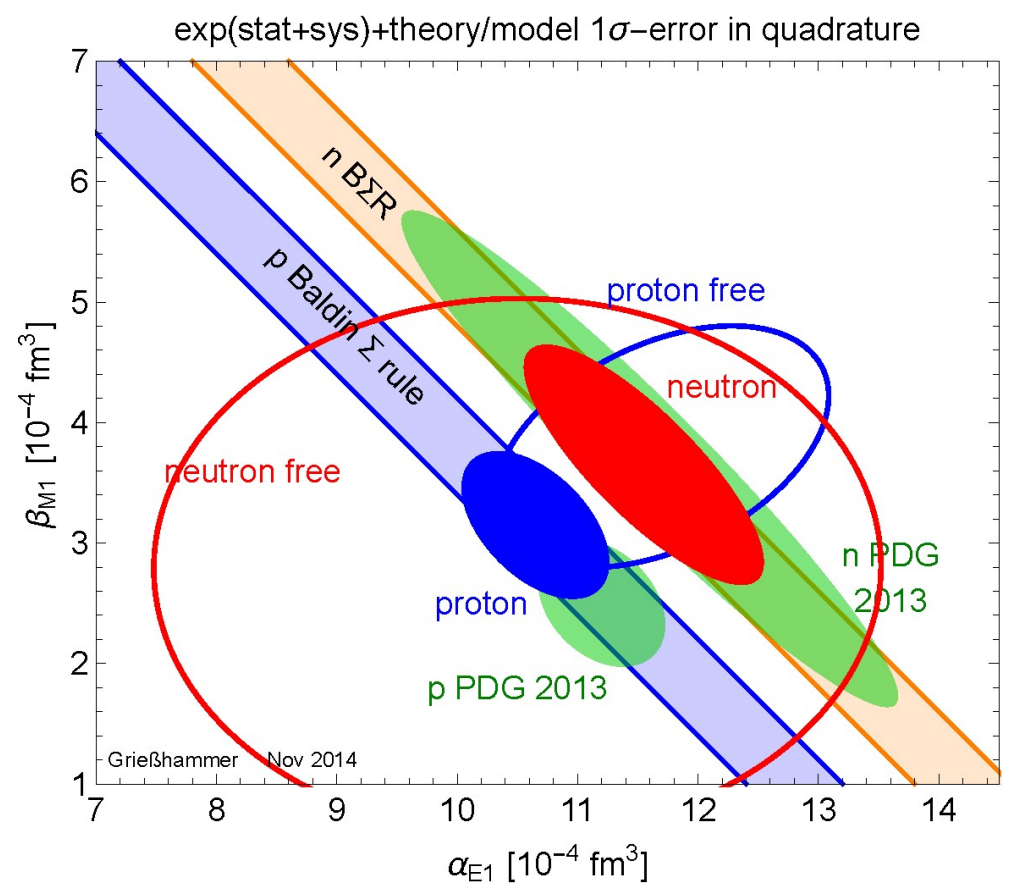

Figure 2: World data for $\alpha$ and $\beta$ of proton and neutron [39].

Results concerning neutron spin polarizabilities are scarce. The neutron spin polarizability $\gamma_{\pi}^{(n)}$ was only directly measured by quasi-free Compton scattering from deuteron (Mainz): $\gamma_{\pi}^{(n)}=$ $(58.6 \pm 4.0) \times 10^{-4} \mathrm{fm}^{4}[40,41]$. In addition, $\gamma_{0}^{(n)} \approx-0.38 \times 10^{-4} \mathrm{fm}^{4}$ was extracted from VPIFA93 multipole analysis (not a direct measurement) [43].

\subsection{Compton experiments at $\mathrm{HI} \gamma \mathrm{S}$ Facility}

The Compton Scattering experiment on unpolarized liquid hydrogen target [42] at $\mathrm{HI} \gamma \mathrm{S}$ will be the first measurement of $\alpha_{p}$ and $\beta_{p}$ (subscript $\mathrm{p}$ for proton) without using the Baldin Sum-Rule, and will have the same level of accuracy as of extraction based on the sum rule from previous experiments. The theoretical study of Compton scattering off proton targets using effective field theory in addition to Eq. (2) is presented in [44], which serves as the theoretical basis of the Baldin Sum-Rule independent extraction of $\alpha_{p}$ and $\beta_{p}$ using linearly polarized $\gamma$ beams. In this experiment linearly polarized $\gamma$ beam at $85 \mathrm{MeV}$ beam energy scattered from the target will be detected by the HI $\gamma \mathrm{S}$ NaI Detector Array (HINDA).

The Compton Scattering experiment on unpolarized liquid deuteron target [42] at HI $\gamma \mathrm{S}$ will enable the EFT extraction of $\beta_{n}$ to better than $20 \%$ uncertainty, while the current extraction is at a level of $40 \%$ which is dominated by the statistical uncertainty [38]. The extraction of $\alpha_{n}$ and $\beta_{n}$ from Compton Scattering data on deuteron target with input of proton electromagnetic polarizabilities from other experiments is presented in [38] and references therein. In this experiment, circularly polarized $\gamma$ beam at 65 and $100 \mathrm{MeV}$ will be scattered from unpolarized liquid deuteron target. The scattered photons will be detected by HINDA. 
The Double-polarized Compton Scattering experiment at $\mathrm{HI} \gamma \mathrm{S}$ will provide access to three of the four neutron spin polarizabilities $\left(\gamma_{1}, \gamma_{2}\right.$ and $\left.\gamma_{4}\right)$, by measuring spin-dependent differential cross sections from elastic Compton scattering of circularly polarized photons on a high-pressure polarized ${ }^{3} \mathrm{He}$ target. Favorable photon beam conditions include a photon energy above $120 \mathrm{MeV}$, a minimum photon flux of $5 \times 10^{7} \gamma / \mathrm{sec}$ and photon energy spread of less than 3.0\%. Photons scattered from the ${ }^{3} \mathrm{He}$ target will be detected by HINDA. Sensivity to the $\gamma$ 's is provided by forming the spin dependent differential cross section differences.

The spin dependent differential cross section differences $\Delta_{z}$ and $\Delta_{x}$ correspond to target spin being parallel and perpendicular to the circularly polarized photon beam, respectively.

$$
\begin{aligned}
& \Delta_{z}=\frac{d \sigma_{\uparrow \uparrow}}{d \Omega}-\frac{d \sigma_{\uparrow \downarrow}}{d \Omega} \\
& \Delta_{x}=\frac{d \sigma_{\uparrow \rightarrow}}{d \Omega}-\frac{d \sigma_{\uparrow \leftarrow}}{d \Omega}
\end{aligned}
$$

The first arrow in each differential cross section (DCS) $\frac{d \sigma}{d \Omega}$ corresponds to the photon beam polarization (along its momentum direction). The second arrow corresponds to the direction of target polarization. The DCS for nucleon target with certain beam and target polarization can be calculated using the amplitude as in Eq. (2), while the much more complicated ${ }^{3} \mathrm{He}$ case has been calculated by D. Shukla using chiral perturbation theory $(\chi \mathrm{PT})[35,36,37]$. The sensitivity of $\Delta_{z}$ to neutron spin polarizability $\gamma_{1}$ is shown in Fig. $3[35,36,37]$. Experimentally measured $\Delta_{z}$ (simulated $\Delta_{z}$ at three scattering angles are shown in Fig. 3 as an example) will be fitted to the theoretical calculations by setting spin polarizabilities as fit parameters (Fig. 3).

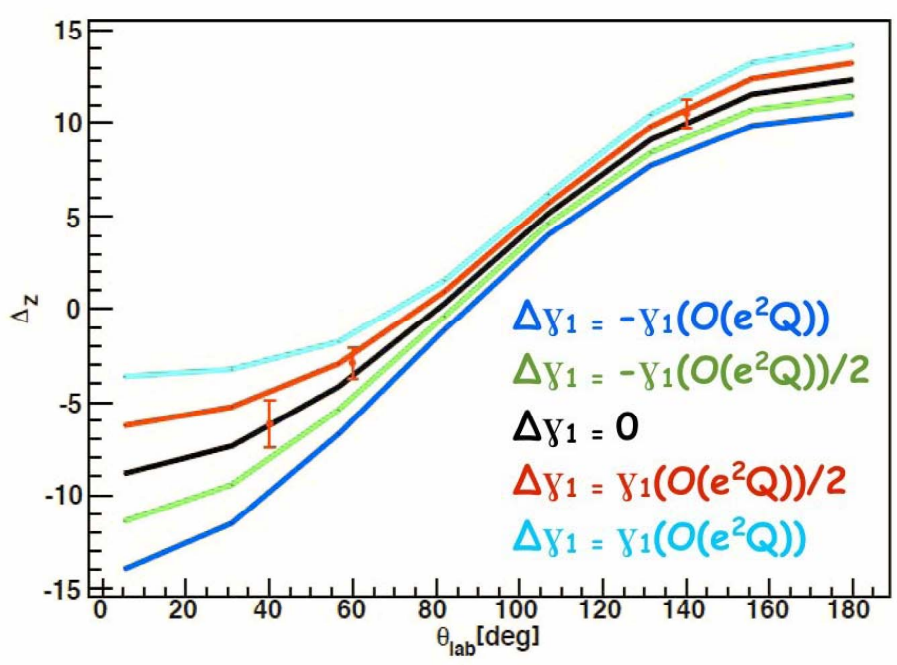

Figure 3: Calculated asymmetry $\Delta_{z}$ (in unit of nb/sr) at $120 \mathrm{MeV}$ beam energy [35, 36, 37]. Different curves correspond to varying $\gamma_{1}$ by cerntain amounts $\left(\Delta \gamma_{1}\right)$. 


\section{Acknowledgment}

The author wish to thank A. Deltuva, A.C. Fonseca, J. Golak, P. U. Sauer, R. Skibiński and H. Witała for providing theoretical calculations for the photodisintegration of ${ }^{3} \mathrm{He}$, the Duke capture group and in particular M.W. Ahmed for his technical help, H.J. Karwowski for his helpful comments and suggestions, Michael Souza of Princeton University for making the target cells, Gordon Cates' group from University of Virginia for providing the $\mathrm{Rb} / \mathrm{K}$ ampules and for Sol-Gel coating of the target cells, T. Averett for filling the cells, D. Dutta for his help during the data taking of the three-body experiments and Y.K. Wu and the TUNL personnel for the technical support of the experiments. This work is supported by the U.S. Department of Energy under contract number DE-FG02-03ER41231 and Duke University.

\section{References}

[1] S.D. Drell and A.C. Hearn et al. Phys. Rev. Lett. 16, 908 (1966); S.B. Gerasimov, Yad. Fiz. 2, 598 (1965) (Sov. J. Nucl. Phys. 1, 430 (1966)).

[2] H. Dutz et al., Phys. Rev. Lett. 93, 032003 (2004) and references therein.

[3] H. Dutz et al., Phys. Rev. Lett. 94, 162001 (2005) and references therein.

[4] K. Slifer et al. Phys. Rev. Lett. 101, 022303 (2008).

[5] M.W. Ahmed, M.A. Blackston, B.A. Perdue, W. Tornow, H.R. Weller, B. Norum, B. Sawatzky, R.M. Prior, and M.C. Spraker, Phys. Rev. C 77, 044005 (2008).

[6] J. Ahrens et al., Phys. Lett. B 672, 328 (2009) and references therein.

[7] G. Laskaris et al., Phys. Rev. Lett. 110, 202501 (2013); Phys. Rev. C 89, 024002 (2014).

[8] G. Laskaris Ph.D.thesis, Duke University, 2015; G. Laskaris et al., Measurement of the Doubly-Polarized ${ }^{3} \vec{H} e(\vec{\gamma}, n) p p$ Reaction at $16.5 \mathrm{MeV}$ and Its Implications for the GDH Sum Rule, submitted to Phys. Rev. Lett., arXiv:1506.00332.

[9] P. Aguar Bartolomé et al., Phys. Lett. B 723, 71 (2013).

[10] S. Costanza et al., Eur. Phys. J. A 50, 173 (2014).

[11] H. Gao, W. Chen, X. Zong, Proc. Sci., CD09 (2009), 101.

[12] H. Gao et al. Phys. Rev. C 50, R546 (1994).

[13] W. Xu et al. Phys. Rev. Lett. 85, 2900 (2000).

[14] S. Riordan et al., Phys. Rev. Lett. 105, 262302 (2010).

[15] P.L. Anthony et al. Phys. Rev. D 54, 6620 (1996).

[16] J.L. Friar, B.F. Gibson, G.L. Payne, A.M. Bernstein, and T.E. Chupp, Phys. Rev. C 42, 2310 (1990).

[17] L.D. Faddeev, Zh. Eksp. Theor. Fiz. 391459 (1960); Sov. Phys. JETP 12, 1041 (1961).

[18] E.O. Alt, P. Grassberger, and W. Sandhas, Nucl. Phys. B2, 167 (1967).

[19] A. Deltuva,L. P. Yuan,J. Adam,A. C. Fonseca,P. U. Sauer, Phys. Rev. C 69, 034004 (2004); A. Deltuva, A.C. Fonseca, and P.U. Sauer, Phys. Rev. C 72, 054004 (2005); Ann. Rev. Nucl. Part. Sci. 58, 27 (2008); A. Deltuva, A.C. Fonseca, and P.U. Sauer, Phys. Rev. C 80, 064004 (2009). 
[20] R. Skibiński, J. Golak, H. Witała, W. Glöckle, A. Nogga, and H. Kamada, Phys. Rev. C 72, 044002 (2005); R. Skibiński, J. Golak, H. Witała, W. Glöckle, H. Kamada and A. Nogga, Phys. Rev. C 67, 054002 (2003); R. Skibiński, J. Golak, H. Kamada, H. Witała, W. Glöckle and A. Nogga, Phys. Rev. C 67, 054001 (2003).

[21] R. B Wiringa, V. G. J. Stoks, R. Schiavilla. Phys. Rev. C 51, 38 (1995).

[22] R. Machleidt and K. Holinde and Ch. Elster, Phys. Rep. 149, 1 (1987).

[23] R. Machleidt, Phys. Rev. C 63, 024001 (2001).

[24] J. Carlson, V.R. Pandharipande, and R.B. Wiringa, Nucl. Phys. A 401, 59 (1983).

[25] H.R. Weller, M.W. Ahmed, H. Gao, W. Tornow, Y.K. Wu, M. Gai, and R. Miskimen, Prog. Part. Nucl. Phys. 62, 257 (2008).

[26] D.M. Skopik, Y.M. Shin, M.C. Phenneger, and J.J. Murphy II, Phys. Rev. C 9, 531 (1974);

Y. Birenbaum, S. Kahane, and R. Moreh, Phys. Rev. C 32, 1825 (1985); R. Bernabei et al., Phys. Rev. Lett. 57, 1542 (1986); A. De Graeve et al., Phys. Rev. C 45, 860 (1992).

[27] W. Happer Rev. Mod. Phys. 42, 169 (1972).

[28] K. Kramer, X. Zong, R. Lu, D. Dutta, H. Gao, X. Qian, Q. Ye, X. Zhu, T. Averett, and S. Fuchs, Nucl. Instrum. Methods Phys. Res., Sect. A 582, 318 (2007).

[29] Q. Ye, G. Laskaris, W. Chen, H. Gao, W. Zheng, X. Zong, T. Averett, G.D. Cates, and W.A. Tobias, Eur. Phys. J. A 44, 55 (2010).

[30] W. Lorenzon, T.R. Gentile, H. Gao, R.D. McKeown, Phys. Rev. A 47, 468 (1993).

[31] M.V. Romalis and G.D. Cates, Phys. Rev. A 58, 3004 (1998), and the references therein.

[32] S. Agostinelli et al., Nucl. Inst. Meth. A 506, 250 (2003).

[33] S. Ragusa, Phys. Rev. D 47, 3757 (1993).

[34] H.W. Grie $\beta$ hammer et al., Progress in Particle and Nuclear Physics 67, 841 (2012).

[35] D. Shukla et al., Nuclear Physics A 819, 98 (2009).

[36] D. Choudhury et al., Phys. Rev. Lett. 98, 232303 (2007)

[37] D. Choudhury, Ph. D. thesis, Ohio University (2006).

[38] L.S. Myers et al., Phys. Rev. Lett. 113, 262506 (2014).

[39] H.W. Grie $\beta$ hammer, private communication (2014).

[40] M. Schumacher. Prog. Part. Nucl. Phys. 55, 567 (2005).

[41] K. Kossert et al., Phys. Rev. Lett. 88, 162301 (2002) ; Eur. Phys. J. A 16, 259 (2003).

[42] M.W. Ahmed et al., proposals submitted to HI $\gamma \mathrm{S}$ PAC and private communication.

[43] A.M. Sandorfi et al., Phys. Rev. D 50 (R), 6681 (1994).

[44] V. Lensky et al., Eur. Phys. J. C 65195209 (2010). 